\title{
MATHEMATIKA
}

A JOURNAL OF PURE AND APPLIED MATHEMATICS

Vol. 29. Part 2.

December 1982.

No. 58

\section{FURTHER LATTICE PACKINGS IN HIGH DIMENSIONS}

\section{A. BOS, J. H. CONWAY AND N. J. A. SLOANE}

Abstract. Barnes and Sloane recently described a "general construction" for lattice packings of equal spheres in Euclidean space. In the present paper we simplify and further generalize their construction, and make it suitable for iteration. As a result we obtain lattice packings in $\mathbb{R}^{m}$ with density $\Delta$ satisfying $\log _{2} \Delta \sim-m \log _{2}^{*} m$, as $m \rightarrow \infty$, where $\log _{2}^{*} m$ is the smallest value of $k$ for which the $k$-th iterated logarithm of $m$ is less than 1 . These appear to be the densest lattices that have been explicitly constructed in high-dimensional space. New records are also established in a number of lower dimensions, beginning in dimension 96.

§1. Introduction. In the past 23 years a series of papers $[1-3,5,8-12,15-17]$ have described a variety of methods for packing equal spheres in Euclidean space. The present paper continues the sequence by simplifying the "general construction" of Barnes and Sloane [1], and thereby eliminating the requirement that the initial lattice be generated by its minimal vectors. This enables us to iterate the construction, obtaining lattice packings in $\mathbb{R}^{m}$ with density $\Delta$ satisfying

$$
\log _{2} \Delta \sim-m \log _{2}^{*} m, \quad \text { as } m \rightarrow \infty,
$$

where $\log _{2}^{*} m$ is the smallest value of $k$ for which the $k$-th iterated logarithm of $m$ is less than 1 . These appear to be the densest lattice packings that have been explicitly constructed in high-dimensional space. Non-lattice packings with $\log _{2} \Delta>-6 m+o(m)$ were constructed in [15] (and non-lattice packings satisfying (1) in [3]), but there is still room for improvement since the best lattice or non-lattice packings are known to lie in the range

$$
-m<\log _{2} \Delta<-0.599 m+o(m), \quad \text { as } m \rightarrow \infty
$$

(see $[4,7,14,18]$ ). The new construction also generalizes that given in $[1]$ so as to use codes over other alphabets.

The construction is described in Section 2, and Section 3 contains a number of applications. A selection of the best packings known in dimensions up to $2^{20}$ will be found in Table 1. 
Notation. The norm of a vector $x$ is its squared length $x . x$. If $L_{m}$ is a lattice in $\mathbb{R}^{m}$ (usually the subscript indicates the dimension), its minimum norm $M$ is $\min \left\{x . x: x \in L_{m}, x \neq 0\right\}$, its determinant $\operatorname{det} L_{m}$ is the volume of a fundamental region, and its density $\Delta$ and centre density $\delta$ are given by

$$
\Delta=\frac{V_{m}(\sqrt{ } M / 2)^{m}}{\operatorname{det} L_{m}}, \quad \text { and } \delta=\frac{\Delta}{V_{m}}
$$

where $V_{m}$ is the volume of an $m$-dimensional sphere of unit radius. In Section 3 we also use the parameters

$$
\mu=\frac{1}{2} m, \quad \gamma=\log _{2} \delta \text {. }
$$

All the packings mentioned in this paper are lattice packings.

§2. The Construction. The ingredients for the construction are a lattice $\Lambda$ in $\mathbb{R}^{m}$, an endomorphism $D$ of $\Lambda$ that satisfies certain conditions, one of which is that $\Lambda / D \Lambda$ is an elementary abelian group $E$ of order $p^{b}$, say, and a family of codes $C_{0} \supseteq C_{1} \supseteq \ldots \supseteq C_{a}$ of length $n$ over $E$; the result is a family $L_{0} \subseteq L_{1} \subseteq \ldots \subseteq L_{a}$ of lattice packings in $\mathbb{R}^{m n}$.

Hypotheses.

(i) Let $\Lambda$ be a lattice in $\mathbb{R}^{m}$ with minimum non-zero norm $M$.

(ii) Let $D$ be an endomorphism of $\Lambda$ which is also a similarity (i.e. a constant times an orthogonal transformation) and which satisfies

$$
p D^{-1}=\sum_{i=0}^{r} \grave{a}_{i} D^{i}
$$

for integers $p \geqslant 1, r \geqslant 0, a_{0}, \ldots, a_{r}$. Let $T=D^{-1}$, so that, from (3), $p T$ is also an endomorphism of $\Lambda$.

(iii) Assume that $\Lambda / D \Lambda$ has the structure of an elementary abelian group $E$ of order $p^{b}$ for some integer $b \geqslant 1$. This implies that there is a $b$-dimensional sublattice $K \subseteq \Lambda$, spanned, say, by vectors $v_{1}, \ldots, v_{b} \in \Lambda$, such that

$$
K /(D \Lambda \cap K) \cong E
$$

The assumptions also imply that

and therefore that

$$
\begin{gathered}
p K \subseteq K \subseteq \Lambda, \\
p K \subseteq p \Lambda \subseteq D \Lambda \subseteq \Lambda,
\end{gathered}
$$

$$
K / p K=K /(D \Lambda \cap K) \cong E .
$$

Note that (4) and (5) imply $p K \subseteq D \Lambda \cap K$, so $K / p K \supseteq K /(D \Lambda \cap K)$. But 
both sides have order $p^{b}$ and so must be equal, and (6) follows. Furthermore

$$
|\operatorname{det} T|=\frac{1}{p^{b}}=t^{m}, \text { say , }
$$

and $T$ multiplies norms by $t^{2}$.

(iv) Assume that all $p^{b}-1$ non-zero congruence classes of $T \Lambda / \Lambda$ have minimum norm at least $t^{2} M$.

(v) Let $\phi$ denote the natural map from $\mathbb{Z} / p \mathbb{Z}$ to $\mathbb{Z}$ which takes the congruence class $\bar{x}$ to $x$, for $x \in\{0,1, \ldots, p-1\}$. The elements of $E$ may be identified with the $b$-tuples $\bar{X}=\left(\bar{x}_{1}, \ldots, \bar{x}_{b}\right)$, where all $\bar{x}_{i} \in \mathbb{Z} / p \mathbb{Z}$. Then

$$
\begin{aligned}
\bar{X} \rightarrow V(\bar{X}) & =\phi\left(\bar{x}_{1}\right) v_{1}+\ldots+\phi\left(\bar{x}_{b}\right) v_{b} \\
& =x_{1} v_{1}+\ldots+x_{b} v_{b}
\end{aligned}
$$

is a map from $E$ into $\Lambda$, and

$$
\overline{\mathbf{X}}=\left(\bar{X}_{1}, \ldots, \bar{X}_{n}\right) \rightarrow V(\overline{\mathbf{X}})=\left(V\left(\bar{X}_{1}\right), \ldots, V\left(\bar{X}_{n}\right)\right)
$$

maps $E^{n}$ into $\Lambda^{n}$.

(vi) Let $C_{0} \supseteq C_{1} \supseteq \ldots \supseteq C_{a}$ be additive codes $\dagger$ over $E$ of length $n$, where $C_{i}$ contains $p^{b k_{i}}$ codewords and has minimum distance $d_{i}$ (we indicate this by saying that $C_{i}$ has parameters $\left[n, k_{i}, d_{i}\right]$ ), and suppose that $C_{0}$ is the trivial $[n, n, 1]$ code. Let $\mathbf{c}_{1}, \ldots, \mathbf{c}_{b k_{a}} \in E^{n}$ be chosen so that a typical codeword of $C_{i}$ can be written as

for $i=1, \ldots, a$.

$$
\sum_{j=1}^{b k_{i}} \bar{x}_{j} \mathbf{c}_{j}, \quad \bar{x}_{j} \in \mathbb{Z} / p \mathbb{Z},
$$

The New Lattices. Let $L_{0}=\Lambda^{n}$ and, for $i=1,2, \ldots, a$, define

$$
L_{i}=\bigcup_{x_{1}, \ldots, x_{b k_{i}}}\left\{L_{i-1}+\sum_{j=1}^{b k_{i}} x_{j} T^{i} V\left(\mathbf{c}_{j}\right)\right\},
$$

where $x_{1}, \ldots, x_{b k_{i}} \in\{0,1, \ldots, p-1\}$. By abuse of notation we shall also use $D$ and $T$ to denote the maps $(D, D, \ldots, D)$ and $(T, T, \ldots, T)$ acting on $\mathbb{R}^{m n}$. It is clear that $L_{0}$ is a lattice and that $D$ and $p T$ are endomorphisms of $L_{0}$.

THEOREM 1. For $i=1, \ldots, a$,

(a) $L_{i}$ is a lattice, and in fact

$$
L_{i}=\mathbb{Z}\left\langle L_{i-1}, T^{i} V\left(\mathbf{c}_{1}\right), \ldots, T^{i} V\left(\mathbf{c}_{b k_{i}}\right)\right\rangle
$$


(b) $D$ maps $L_{i}$ into $L_{i-1}$; and

(c) $p T$ is an endomorphism of $L_{i}$.

Proof. The proof is by induction on $i$, the results for $i=0$ having already been mentioned. (a) To show that $L_{i}$ is a lattice, we write any integer combination of the elements on the right-hand side of $(8)$ as

$$
l+p \sum_{j=1}^{b k_{i}} y_{j} T^{i} V\left(\mathbf{c}_{j}\right)+\sum_{j=1}^{b k_{i}} z_{j} T^{i} V\left(\mathbf{c}_{j}\right),
$$

where $l \in L_{i-1}$ and $0 \leqslant z_{j} \leqslant p-1$. But

$$
p T^{i} V\left(\mathbf{c}_{j}\right)=p T \cdot T^{i-1} V\left(\mathbf{c}_{j}\right) \in L_{i-1}
$$

by the induction hypothesis, so (10) becomes

$$
l^{\prime}+\sum_{j=1}^{b k_{i}} z_{j} T^{i} V\left(\mathbf{c}_{j}\right)
$$

where $l^{\prime} \in L_{i-1}$, which by (8) is in $L_{i}$. Thus $L_{i}$ is a lattice, and therefore can be defined by the right-hand side of (9). Now (b) follows from

$$
D T^{i} V\left(\mathbf{c}_{j}\right)=T^{i-1} V\left(\mathbf{c}_{j}\right) \in L_{i-1}
$$

From (3) and (b) we have

which is (c).

$$
p T\left(L_{i}\right)=p D^{-1}\left(L_{i}\right)=\sum_{j=0}^{r} a_{j} D^{j}\left(L_{i}\right) \subseteq L_{i},
$$

TheOREM 2. For $i=0,1, \ldots, a$, the determinant, minimum norm and centre density of $L_{i}$ are given by

$$
\begin{gathered}
\operatorname{det} L_{i}=\frac{(\operatorname{det} \Lambda)^{n}}{b \sum_{j=1}^{i} k_{j}}, \\
\bar{M}=\min \left\{M, d_{j} t^{2 j} M \text { for } j=1, \ldots, i\right\},
\end{gathered}
$$

and

$$
\delta=\bar{M}^{m n / 2} / 2^{m n} \operatorname{det} L_{i}
$$

respectively.

Proof. Equation (11) is immediate from (8), and (12) follows from the definition of $L_{i}$ and the fact that the minimum distance of $C_{i}$ is $d_{i}$. For more details compare the proof of Theorem 3 in [1].

Usually we are only interested in the finest lattice $L_{a}$. The construction may now be applied to $L_{a}$, since it inherits $D$ and $T$ from $\Lambda$, and (3) still holds. The values of $p$ and $t$ are unchanged, while $b$ becomes $n b$. 
\$3. Examples. The above construction includes some of the most important special cases of Constructions $A$ and $B$ of [12] (see [1]), as well as the constructions of $[1]$, and so a large number of examples are already known. In this section we give several additional examples of some interest.

If $D$ is a norm-doubler. In all these examples $D$ will be a norm-doubler, so that $t=1 / \sqrt{ } 2, p=2$ and $b=m / 2$. We shall take the codes $C_{i}, i \geqslant 1$, to be maximal distance separable codes over the field $G F\left(2^{b}\right)$, with parameters $\left[n, k_{i}=n-2^{i}+1, d_{i}=2^{i}\right]$. In general, the largest $n$ for which such codes are presently known to exist is $2^{b}+1$ (see [13, Chapter 11]). When $n=2^{b}+1, C_{i}, i \geqslant 1$, can be taken to be the cyclic code with generator polynomial

$$
g(x)=\prod_{j=-s}^{s}\left(x+\xi^{j}\right)
$$

where $\xi \in G F\left(2^{2 b}\right)$ is a primitive $n$-th root of unity and $s=2^{i-1}-1$ (see the proof of Theorem 9, Chapter 11 of [13])†. Besides being additive, these codes are also closed under multiplication by elements of $G F\left(2^{b}\right)$. However we make no use of this multiplicative structure in our construction. Clearly $C_{0} \supseteq C_{1} \supseteq C_{2} \supseteq \ldots$. For $n$ less than $2^{b}+1$ the codes are shortened by setting the appropriate number of information symbols equal to zero. If $n$ lies in the range $2 \leqslant n \leqslant 2^{b+1}$, we use the codes $C_{1}, C_{2}, \ldots, C_{a}$, where $a$ is determined by $2^{a} \leqslant n<2^{a+1}$. Then the value of $\sum_{j=1}^{a} k_{j}$ for use in (11) is

$$
a n-2^{a+1}+a+2 .
$$

Since $d_{j} t^{2 j}=1$, the minimum norm is unchanged (see (12)). This version of the construction therefore converts a lattice $\Lambda$ in $\mathbb{R}^{m}$ with centre density $\delta=2^{\gamma}$ (say) into a lattice $\Lambda^{\prime}=L_{a}$ in $\mathbb{R}^{m n}$ with centre density $\delta^{\prime}=2^{\gamma^{\prime}}$, where from (11), (13), (14)

$$
\gamma^{\prime}=n \gamma+\frac{m}{2}\left(a n-2^{a+1}+a+2\right)
$$

Our examples are all descendants of the three lattices $\mathbb{Z}^{2}, \Lambda_{24}$ and $\mathbb{P} 48 q$.

Packings constructed from the Lattice $\mathbb{Z}^{2}$. For the first example we take $\Lambda$ to be the familiar two-dimensional square lattice $\mathbb{Z}^{2}$ with minimum norm $M=1$ (see Figure 1). Although this is not a particularly dense packing, it has surprisingly good progeny. We let $D$ map $(1,0)$ to $(1,1)$, and $(0,1)$ to $(1,-1)$, or in matrix notation, with $D$ mapping $v$ to $v D$,

$$
D=\left(\begin{array}{rr}
1 & 1 \\
1 & -1
\end{array}\right), \quad T=D^{-1}=\frac{1}{2}\left(\begin{array}{rr}
1 & 1 \\
1 & -1
\end{array}\right),
$$

so that (Eq. 3) reads $2 D^{-1}=D$. Then $\Lambda / D \Lambda \cong \mathbb{Z} / 2 \mathbb{Z}, p^{b}=2^{1}$, and we may take $K$ to be the 1-dimensional lattice spanned by $v_{1}=(1,0)$ (see Figure 1 ).

† That theorem is incorrect if the field size $q$ is odd (see $[13, \mathrm{p} . \mathrm{xii}]$ ) but that does not concern us since here $q$ is even. 


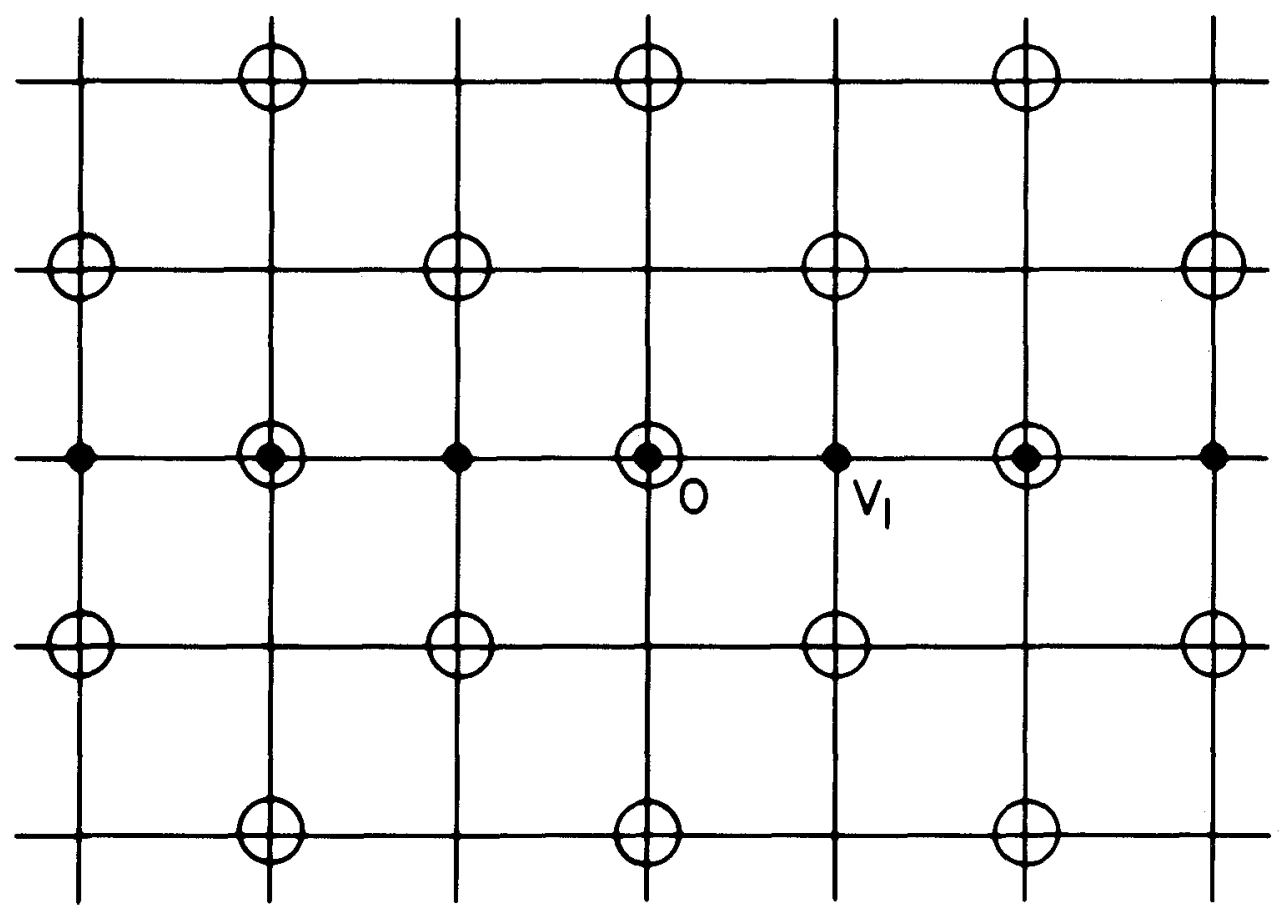

Figure 1. The lattice $\Lambda=\mathbb{Z}^{2}$ (represented by the underlying grid of points), and the sublattices $D \Lambda$ (small circles) and $K$ (solid circles).

We apply our construction to $\mathbb{Z}^{2}$, with $n=2$ and $C_{1}$ equal to the code $\{00,11\}$ over $G F(2)$, and obtain the Schläfli lattice $D_{4}$, with $\gamma=-3$ (from (15)).

From now on we need not specify $D, T$ or $p^{b}$, since these are determined (see the remarks at the end of Section 2). Applying the construction to $D_{4}$ with $n=1, \ldots, 5$ we obtain the laminated lattices (see [5])

for which $\gamma$ is

$$
\Lambda_{4}=D_{4}, \quad \Lambda_{8}=E_{8}, \quad \Lambda_{12}, \quad \Lambda_{16}, \quad \Lambda_{20}
$$

respectively.

$$
-3,-4,-5,-4,-3
$$

$E_{8}$ (the first grandchild of $\mathbb{Z}^{2}$ ) was one of the examples studied in [1]†. It produces $\Lambda_{16}$ again, packings $\bar{\Lambda}_{32}$ and $\bar{\Lambda}_{40}$ with the same density as the laminated lattice $\Lambda_{32}$ and $\Lambda_{40}$, excellent packings in dimensions 80 to 136 , but indifferent packings in dimensions 24 and 48 to 72 .

Table 1 contains a selection of the best lattice packings known up to dimension $2^{20}$. The second column gives $\gamma=\log _{2} \delta$, while a parenthesized entry $(\Lambda)$ in the third column indicates that the packing can be obtained by applying our construction to the lattice $\Lambda$. Those packings (in dimension $\geqslant 96$ ) for which no reference is given are believed to set new records for lattices.

The children of $E_{8}$ extend to dimension 136 in the table and then stop. Beyond this point the lattices obtained by applying the construction to $\Lambda_{12}$ and $\Lambda_{16}$ are roughly comparable, up to dimension $16\left(2^{8}+1\right)$. The children of $\Lambda_{20}, \bar{\Lambda}_{32}$ and $\bar{\Lambda}_{40}$

† The map $D$ used in [1] differs from the one given here, but is equivalent to it. One can in fact show that there is essentially only one norm-doubling map for $E_{8}$ with the requisite properties. 
Table 1. A selection of the densest known packings $n=$ dimension, $\gamma=\log _{2}$ (centre density)

\begin{tabular}{|c|c|c|c|c|c|c|}
\hline$n$ & $\gamma$ & name & Ref. & $n$ & $\gamma$ & name \\
\hline 0 & 0 & $\Lambda_{0}$ & & 492 & 683 & $\left(\Lambda_{12}\right)$ \\
\hline 4 & -3 & $D_{4}$ & & 496 & 660 & $\left(\Lambda_{16}\right)$ \\
\hline 8 & -4 & $E_{8}$ & & 500 & 665 & $\left(\Lambda_{20}\right)$ \\
\hline 16 & -4 & $\Lambda_{16}^{\circ}$ & [2] & 504 & 708 & $\left(\Lambda_{12}\right)$ \\
\hline 24 & 0 & $\Lambda_{24}$ & [9] & 512 & 698 & $B_{512}$ \\
\hline 32 & 0 & $\Lambda_{32}$ & {$[2,12]$} & 516 & 733 & $\left(\Lambda_{12}\right)$ \\
\hline 40 & 4 & $\Lambda_{40}$ & [12] & 1024 & 1856 & $\left(\Lambda_{16}\right)$ \\
\hline 48 & 14.039 & $\mathbb{P} 48 q$ & {$[12]$} & 1032 & 1896 & $\left(\Lambda_{24}\right)$ \\
\hline 64 & 22 & - & {$[1]$} & 1040 & 1900 & $\left(\Lambda_{16}\right)$ \\
\hline 80 & 36 & $\left(E_{8}\right)$ & [1] & 2040 & 4680 & $\left(\Lambda_{24}\right)$ \\
\hline 96 & 52.078 & (P48q) & & 2048 & 4680 & $\left(\Lambda_{16}\right)$ \\
\hline 128 & 88 & $\left(E_{8}\right)$ & [1] & 2064 & 4752 & $\left(\Lambda_{24}\right)$ \\
\hline 136 & 100 & $\left(E_{8}\right)$ & [1] & 4096 & 11344 & $\left(\Lambda_{16}\right)$ \\
\hline 160 & 112 & $\left(\Lambda_{16}\right)$ & & 4104 & 11400 & $\left(\Lambda_{24}\right)$ \\
\hline 168 & 120 & $\left(\Lambda_{24}\right)$ & [1] & 8208 & 26808 & $\left(\Lambda_{24}\right)$ \\
\hline 176 & 132 & $\left(\Lambda_{16}\right)$ & & 16392 & 61608 & $\left(\Lambda_{24}\right)$ \\
\hline 192 & 156 & $\left(\Lambda_{24}\right)$ & [1] & 32784 & 139488 & $\left(\Lambda_{24}\right)$ \\
\hline 256 & 250 & $B_{256}$ & [1] & 65544 & 311496 & $\left(\Lambda_{24}\right)$ \\
\hline 264 & 264 & $\left(\Lambda_{24}\right)$ & [1] & 131088 & $6.613 \times 10^{5}$ & $(\mathbb{P} 48 q)$ \\
\hline 272 & 268 & $\left(\Lambda_{16}\right)$ & & 262176 & $1.453 \times 10^{6}$ & $(\mathbb{P} 48 q)$ \\
\hline 288 & 300 & $\left(\Lambda_{24}\right)$ & [1] & 524304 & $3.168 \times 10^{6}$ & (P48q) \\
\hline 384 & 464 & $\left(\Lambda_{16}\right)$ & & 1048608 & $6.861 \times 10^{6}$ & $(\mathbb{P} 48 q)$ \\
\hline
\end{tabular}

are not quite as good as other known packings. However, examination of Table 1 (and of a more extensive table of which this is an extract) shows that at the moment one cannot completely ignore the children of any of these lattices.

Packings constructed from the Leech lattice $\Lambda_{24}$. The Leech lattice $\Lambda_{24}$ is another example that was studied in [1], using the norm-doubling map $D=I-i$, where $i$ is a certain automorphism of $\Lambda_{24}$. There is a second choice for $D$, essentially different from $I-i$, which may be defined as follows. Let the 24 coordinate positions be labelled $\infty, 0,1, \ldots, 22$, and as usual (see $[9,12]$ ) let $\Lambda_{24}$ be spanned by the vectors:-

$\left(2^{12}, 0^{12}\right), \quad 23$ vectors, supported on a translate of $\{0\} \cup q$, where $q$ denotes the set of non-zero quadratic residues modulo 23 ,

$\left(-3,1^{23}\right)$, a single vector, and

$\left( \pm 4^{2}, 0^{22}\right), \quad 4 \cdot\left(\begin{array}{c}24 \\ 2\end{array}\right)$ vectors.

Then we may take $D$ to be represented by the matrix

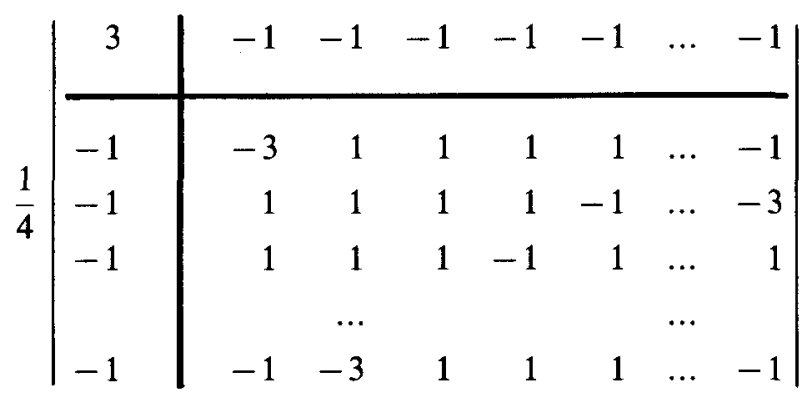


where in the second row there is a -3 in position 0,1 's in the positions in $q$, and -1 's elsewhere. Also $T=D^{-1}=\frac{1}{2} D$ and $p^{b}=2^{12}$. Applying our construction to $\Lambda_{24}$ using either version of $D$ produces lattices which run neck and neck with $\Lambda_{12}$ and $\Lambda_{16}$ up to dimension 4096 , and beyond this point are the best packings known up to dimension $24\left(2^{12}+1\right)$ (see Table 1 and $[1]$ ).

Packings constructed from $\mathbb{P} 48 q$. Our third starting point is the 48-dimensional lattice $P 48 q$ constructed in [12], which has a norm-doubling map very similar to the second map found for $\Lambda_{24}$. If the coordinate positions are labelled $\infty, 0,1, \ldots, 46$, we may take $\mathbb{P} 48 q$ to be spanned by the vectors:-

$\left(2^{24}, 0^{24}\right), \quad 47$ vectors, supported on a translate of $\{0\} \cup q$, where $q$ denotes the set of non-zero quadratic residues modulo 47 ,

$\left(-5,1^{47}\right)$, a single vector, and

$\left( \pm 6^{2}, 0^{46}\right), \quad 4 \cdot\left(\begin{array}{c}48 \\ 2\end{array}\right)$ vectors.

Then we may take $D$ to be

$$
\frac{1}{6}\left|\begin{array}{r|rrrrrrr}
5 & -1 & -1 & -1 & -1 & -1 & \ldots & -1 \\
\hline-1 & -5 & 1 & 1 & 1 & 1 & \ldots & -1 \\
-1 & 1 & 1 & 1 & 1 & -1 & \ldots & -5 \\
-1 & 1 & 1 & 1 & -1 & 1 & \ldots & 1 \\
& & & \ldots & & & \ldots & \\
-1 & -1 & -5 & 1 & 1 & 1 & \ldots & -1
\end{array}\right|
$$

where in the second row there is a -5 in position 0,1 's in the positions in $q$, and -1 's elsewhere. Also $T=D^{-1}=\frac{1}{2} D$. This gives excellent packings in dimensions 96 and from $24\left(2^{12}+1\right)$ to $48\left(2^{24}+1\right)$.

Higher dimensions. The construction may be applied repeatedly to any of these packings, producing an infinite tree of lattices. We may derive a lower bound to the density obtained in this way in high dimensions by the following argument. Let $\Lambda$ be any of the lattices described in this section, with say dimension $m=2 \mu$, centre density $\delta=2^{\gamma}$, and norm-doubling map $D$. We apply our construction using codes of length $n=2^{\mu}$, obtaining a lattice $\Lambda^{\prime}\left(=L_{a}\right)$ in dimension $m^{\prime}=2 \mu^{\prime}$ with centre density $\delta^{\prime}=2^{\gamma^{\prime}}$, where, from (15)

$$
\begin{aligned}
& \mu^{\prime}=\mu 2^{\mu}, \\
& \gamma^{\prime}=2^{\mu} \gamma+\mu(\mu-2) 2^{\mu}+\mu(\mu+2) .
\end{aligned}
$$


It is simpler to work with the density $\Delta$ rather than the centre density $\delta$, so let us define

$$
\eta=-\frac{\log _{2} \Delta}{m}
$$

(We know from (2) that for large dimensions $m$ the best packings satisfy $0.599 \leqslant \eta \leqslant 1$.) Now

$$
\log _{2} \Delta=\log _{2} \delta-\mu \log _{2} \mu+\mu \log _{2}(\pi e)-\frac{1}{2} \log _{2}(2 \pi \mu)+o(1),
$$

and so (17) becomes

$$
\eta^{\prime}=\eta+1-\frac{1}{4 \mu} \log _{2}(2 \pi \mu)+o\left(\frac{1}{\mu}\right)
$$

The solution of (16) and (18) is

$$
\eta(m)=\log _{2}^{*}(m)
$$

and therefore applying the construction repeatedly leads to lattices satisfying (1). Note that this asymptotic behavior is independent of the choice of the initial lattice.

Acknowledgement. We are grateful to Professor E. S. Barnes for some helpful discussions.

\section{References}

1. E. S. Barnes and N. J. A. Sloane. New lattice packings of spheres, Canad. J. Math., to appear.

2. E. S. Barnes and G. E. Wall. Some extreme forms defined in terms of Abelian groups. J. Australian Math. Soc., 1 (1959), 47-63.

3. A. Bos. Sphere packings in high-dimensional space. Preprint.

4. A. Bos. Upper bounds for sphere packings in Euclidean space. IEEE IT Transactions on Information Theory. To appear.

5. J. H. Conway and N. J. A. Sloane. Laminated lattices. Annals of Math., to appear.

6. P. Delsarte. Four fundamental parameters of a code and their combinatorial significance. Information and Control, 23 (1973), 407-438.

7. G. A. Kabatiansky and V. I. Levenshtein. Bounds for packings on a sphere and in space (in Russian). Problemy Peredachi Informatsii, 14 (No, 1, 1978), 3-25. English translation in Problems of Information Transmission, 14 (1978), 1-17.

8. J. Leech. Some sphere packings in higher space. Canad. J. Math., 16 (1964), 657-682.

9. J. Leech. Notes on sphere packings. Canad. J. Math., 19 (1967), 251-267.

10. J. Leech and N. J. A. Sloane. New sphere packings in dimensions 9-15. Bull. Amer. Math. Soc., 76 (1970), 1006-1010.

11. J. Leech and N. J. A. Sloane. New sphere packings in more than 32 dimensions. Proc. Second Chapel Hill Conference on Combinatorial Mathematics and its Applications (Univ. of North Carolina at Chapel Hill, 1970), 345-355.

12. J. Leech and N. J. A. Sloane. Sphere packings and error-correcting codes. Canad. J. Math., 23 (1971), $718-745$.

13. F. J. MacWilliams and N. J. A. Sloane. The Theory of Error-Correcting Codes (North-Holland, Amsterdam, 3rd printing, 1981).

14. C. A. Rogers. Packing and Covering (Cambridge University Press, Cambridge, 1964).

15. N. J. A. Sloane.Sphere packings constructed from BCH and Justesen codes. Mathematika, 19 (1972), 183-190.

16. N. J. A. Sloane. Codes over GF(4) and complex lattices. J. Algebra, 52 (1978), 168-181.

17. N. J. A. Sloane. Self-dual codes and lattices, in Relations Between Combinatorics and Other Parts of Mathematics, Proc. Sympos. Pure Math., 34 (Amer. Math. Soc., Providence, RI, 1979), 273-308 
18. N. J. A. Sloane. Recent bounds for codes, sphere packings and related problems obtained by linear programming and other methods. In Papers in Algebra, Analysis and Statistics, edited by R. Lidl. Contemporary Mathematics, 9 (Amer. Math. Soc., 1982), 153-185.

A. Bos, Corporate ISA,

N.V. Philips' Gloeilampenfabrieken, $5600 \mathrm{MD}$, Eindhoven, Netherlands.

Dr. J. H. Conway,

Department of Pure Mathematics and Mathematical Statistics,

University of Cambridge,

Cambridge. CB2 1SB

Dr. N. J. A. Sloane,

Mathematics and Statistics Research Center, Bell Laboratories,

Murray Hill, NJ 07974, U.S.A.
10E30: NUMBER THEORY; Geometry of Numbers; Lattice packing. 